\title{
SOME INDEFINITE NONLINEAR EIGENVALUE PROBLEMS
}

\author{
A. SUÁREZ* \\ Dpto. Ecuaciones Diferenciales y Análisis Numérico, \\ Fac. Matemáticas, C/ Tarfia s/n, C.P. 41012, \\ Univ. Sevilla, Spain \\ E-mail: suarez@us.es
}

Dedicated to Prof. Jean Mawhin for his first 60 years of Nonlinear Analysis

In this work we study the structure of the set of positive solutions of a nonlinear eigenvalue problem with a weight changing sign. Specifically, the reaction term arises from a population dynamic model. We use mainly bifurcation methods to obtain our results.

\section{Introduction}

The aim of this work is to study some nonlinear indefinite eigenvalue problems of the form

$$
\begin{cases}-\Delta u=\lambda m(x) f(u) & \text { in } \Omega, \\ u=0 & \text { on } \partial \Omega,\end{cases}
$$

where $\Omega \subset \mathbb{R}^{N}$ is a bounded domain with a regular boundary $\partial \Omega, m \in C(\bar{\Omega})$ changes sign, $f$ is a regular function and $\lambda$ plays the role of real parameter. We focus our attention on the case $f(0)=0$ and $\lambda>0$; similar results can be obtained for negative values of $\lambda$.

Depending of the shape of $f$, Eq. (1) models different situations: population dynamics, population genetics, combustion theory,... see [10].

In the linear case, i.e., $f(u)=u,(1)$ is the eigenvalue problem

$$
\begin{cases}-\Delta u=\lambda m(x) u & \text { in } \Omega, \\ u=0 & \text { on } \partial \Omega .\end{cases}
$$

\footnotetext{
*Supported by the Spanish Ministry of Science and Technology under grants BFM2000-
} 0797 and BFM2003-06446. 
It is well known (see for instance [19] and [23]) that there exist two values of $\lambda, \lambda_{-}(m)<0<\lambda_{+}(m)$, called principal eigenvalues because they have associated positive eigenfunctions. In the present work, given $q \in L^{\infty}(\Omega)$ we denote by $\sigma_{1}^{\Omega}[-\Delta+q]$ (we delete the superscript $\Omega$ when no confusion arises) the principal eigenvalue of the problem

$$
-\Delta u+q(x) u=\lambda u \quad \text { in } \Omega, \quad u=0 \quad \text { on } \partial \Omega .
$$

When in (1) the weight does not appear, i.e., $m \equiv 1$, the nonlinear problem

$$
\begin{cases}-\Delta u=\lambda f(u) & \text { in } \Omega, \\ u=0 & \text { on } \partial \Omega,\end{cases}
$$

has been extensively studied. Classical references are [2] and [21], but many others can be given where, as well as existence results, uniqueness ones are shown: [4], [14], [26], [20], [22] and references therein.

Much less is known for problem (1). In [19], assuming for example that $f^{\prime}(0)>0$, the authors showed that there exists an unbounded continuum of positive solutions bifurcating from the trivial solution at $\lambda=\lambda_{+}(m) / f^{\prime}(0)$.

In [8] the authors assumed that $f: I \mapsto \mathbb{R}_{+}, I \subset \mathbb{R}$, and $f^{\prime \prime}<0$ and showed that every positive solution of (1) is stable. If, moreover, $I=[0,1]$, $f(1)=0$ and $f^{\prime}(0)>0$ they proved that there exists a positive solution if, and only if, $\lambda>\lambda_{+}(m) / f^{\prime}(0)$, and in this case the solution is unique. Similar result was shown in [13], although the authors' motivation was to study the problem in the whole space. Very recently, in [9] the authors analyze the particular cases $f(u)=g_{i}(u), i=1,2$ with

$$
g_{1}(u)=u-u^{2}, \quad g_{2}(u)=u+u^{2} .
$$

Observe that the result of [8] can only be applied to $g_{1}$. In [9], without the assumption that $f$ takes only values in $[0,1]$, the main result of $[8]$ was improved showing (by variational method) that, assuming some restriction in the space dimension, there exists positive solution if $\lambda \in\left(0, \lambda_{+}(m)\right)$. For the case, $f=g_{2}$, they also proved the existence of positive solution for $\lambda \in\left(0, \lambda_{+}(m)\right)$ and that there does not exist positive solution at $\lambda=$ $\lambda_{+}(m)$. In [16] these results have been again completed. We prove for $f=g_{1}$ that there exist at least two positive solutions in $\lambda \in\left(\lambda_{+}(m), \infty\right)$, one of them linearly asymptotically stable and that for $f=g_{2}$ there exists positive solution if, and only if, $\lambda \in\left(0, \lambda_{+}(m)\right)$.

In this work, we are going to analyze the following nonlinearities

$$
f_{1}(u)=u-u^{2}-K \frac{u}{1+u}, \quad f_{2}(u)=u+u^{2}-K \frac{u}{1+u},
$$


where $K \in \mathbb{R}$. Observe that the functions in (4) are included in (5). These last nonlinearities arise in population dynamics. Indeed, when $K=0, f_{1}$ is the classical logistic reaction term and for $K \neq 0$ the predation one $K u /(1+u)$ is called the Holling-Tanner term, see for example [7] for an ecological interpretation.

In order to state our main results we need some notations. Specifically, assume that

$$
M_{ \pm}:=\left\{x \in \Omega: m^{ \pm}>0\right\}
$$

are open and regular sets, where $m^{ \pm}$represent the positive and negative part of $m$ respectively; and suppose that $m^{ \pm}(x) \approx\left[\operatorname{dist}\left(x, \partial M_{ \pm}\right)\right]^{\gamma_{ \pm}}$for $x$ close to $\partial M_{ \pm}$and some $\gamma_{ \pm} \geq 0$. The following condition will provide us with a priori bounds of the solutions

$$
2<\min \left\{\frac{N+1+\gamma_{ \pm}}{N-1}, \frac{N+2}{N-2}\right\}
$$

Finally, we define for $K \neq 1$ the values

$$
\lambda_{+}:=\frac{\lambda_{+}(m)}{1-K} \quad \lambda_{-}:=\frac{\lambda_{-}(m)}{1-K},
$$

and $\Pi: \mathbb{R} \times C(\bar{\Omega}) \mapsto \mathbb{R}$ the projection map onto $\mathbb{R}$, i.e. $\Pi(\mu, u)=\mu$. The main results are:

Theorem 1.1. Assume that $K \neq 1$ and (6).

(1) There exists an unbounded continuum $\mathcal{C}$ of positive solutions of (1) bifurcating from the trivial solution at $\lambda=\lambda_{+}$if $K<1$ and $\lambda=\lambda_{-}$ if $K>1$.

(2) The bifurcation is supercritical for $f=f_{1}$ and for $f=f_{2}$ and $K<-1$ or $K>1$ and subcritical for $f=f_{2}$ and $K \in[-1,1)$.

(3) If $f=f_{1}$ and $K<1$ (resp. $f=f_{2}$ and $K>1$ ), then $\Pi(\mathcal{C})=$ $\left(\lambda_{+}, \infty\right)$ (resp. $\left.\left(\lambda_{-}, \infty\right)\right)$. Moreover, if $\left(\lambda, u_{\lambda}\right) \in \mathcal{C}$, then $u_{\lambda}$ is linearly asymptotically and such that $u_{\lambda} \leq \sqrt{1-K}$ (resp. $\left.\sqrt{K}-1\right)$. Furthermore, there exists another positive solution $v_{\lambda}$ for all $\lambda>0$.

(4) If $f=f_{1}$ and $K>1$ (resp. $f=f_{2}$ and $K<-1$ ) then $\Pi(\mathcal{C})=$ $\left(0, \lambda_{*}\right]$ for $\lambda_{*}>\lambda_{-}$(resp. $\left.\lambda_{+}\right)$. Moreover, there exist $\lambda_{0}$ and $\lambda^{*}$ with $\lambda_{0}<\lambda^{*}$ such that for $\lambda \geq \lambda^{*}$ the problem (1) does not admit positive solutions and it possesses at least two positive solutions for $\lambda \in\left(\lambda_{-}, \lambda_{0}\right)$ (resp. $\left.\left(\lambda_{+}, \lambda_{0}\right)\right)$.

(5) If $f=f_{2}$ and $K \in[-1,1)$ there exists positive solution for $\lambda \in$ $\left(0, \lambda_{+}\right)$and (1) does not admit positive solutions for $\lambda \geq \lambda^{*}$. 
(6) In any case, if there exists a solution $v_{\lambda}$ for $\lambda>0$, then $\lim _{\lambda \rightarrow 0}\left\|v_{\lambda}\right\|_{\infty}=+\infty$.

Theorem 1.2. Assume $K=1$ and (6). Then there exists at least a solution $u_{\lambda}$ for $\lambda>0$ and $\lim _{\lambda \rightarrow 0}\left\|u_{\lambda}\right\|_{\infty}=+\infty$.

\section{Remark 1.1.}

(1) The existence of $\mathcal{C}$ is true without assuming (6). In the cases (4) and (5) of Theorem 1.1, $\mathcal{C}$ could "go to infinity" in a value $\lambda^{0}$.

(2) In the particular case $f=f_{2}$ and $K=0$, in [16] it was proved using a Picone inequality that (1) possesses a positive solution if, and only if, $\lambda \in\left(0, \lambda_{+}\right)$.

In Figs. 1 and 2 we have summarized these results (the case $f=f_{2}$ and $K=1$ is similar to $f=f_{1}$ and $K=1$ ).

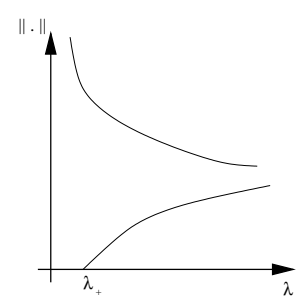

a)

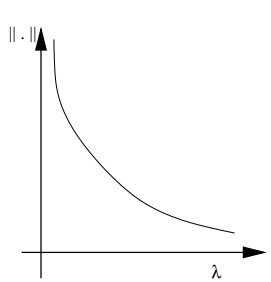

b)

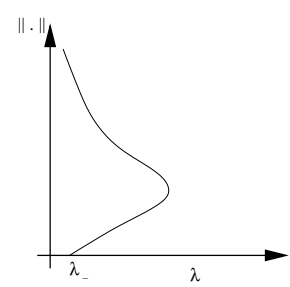

c)

Figure 1. Bifurcation diagrams for $f=f_{1}$ : a) $K<1$; b) $K=1$; c) $K>1$.

The rest of the paper is organized as follows: Secs. 2 and 3 are devoted to prove Theorems 1.1 and 1.2 , respectively.

\section{Proof of Theorem 1.1}

\subsection{Local bifurcation}

In this subsection we show the direction of bifurcation from the trivial solution for both cases $f_{1}$ and $f_{2}$. For that, we write the nonlinearity of the following manner

$$
f(u)=u \mp u^{2}-K \frac{u}{1+u}=u(1-K)+u^{2}\left(\frac{K}{1+u} \mp 1\right) .
$$




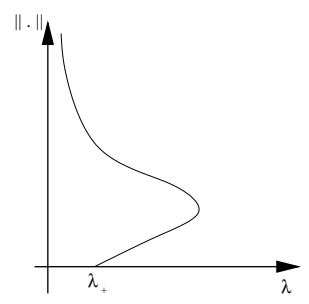

a)

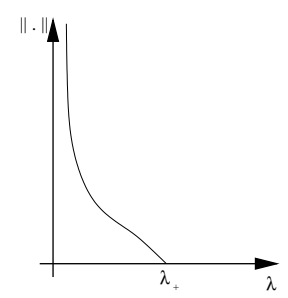

b)

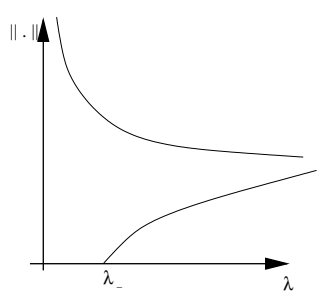

c)

Figure 2. Bifurcation diagrams for $f=f_{2}$ : a) $K<-1$; b) $K \in[-1,1)$; c) $K>1$.

It is clear that to study (1) is equivalent to find zeros of $\mathcal{L}(\lambda) u-N(\lambda, u)=0$, where

$$
\begin{aligned}
& \mathcal{L}(\lambda) u:=u-\lambda(-\Delta)^{-1} m(x)(1-K) u, \\
& N(\lambda, u):=\lambda(-\Delta)^{-1} m(x) u^{2}\left(\frac{K}{1+u} \mp 1\right) .
\end{aligned}
$$

We can prove that

$$
N\left(\mathcal{L}\left(\lambda_{+}\right)\right)=\text {Span }<\varphi^{+}>\quad \text { and } \quad \frac{d}{d \lambda} \mathcal{L}\left(\lambda_{+}\right) \varphi^{+} \notin R\left(\mathcal{L}\left(\lambda_{+}\right)\right)
$$

where, given any linear continuous operator $L, N[L]$ and $R[L]$ stand for the null space and the range of $L$, respectively, and

$$
-\Delta \varphi^{+}=\lambda_{+}(m) m(x) \varphi^{+} \quad \text { in } \Omega, \quad \varphi^{+}=0 \quad \text { on } \partial \Omega .
$$

The first equality of (7) is trivial, for the second expression we need the following result.

Lemma 2.1. For any $p \geq 2$ we have that

$$
\int_{\Omega} m(x)\left(\varphi^{+}\right)^{p}>0
$$

Proof: Multiplying (8) by $\left(\varphi^{+}\right)^{p-1}$ we get

$\lambda_{+}(m) \int_{\Omega} m(x)\left(\varphi^{+}\right)^{p}=\int_{\Omega}\left(-\Delta \varphi^{+}\right)\left(\varphi^{+}\right)^{p-1}=(p-1) \int_{\Omega}\left|\nabla \varphi^{+}\right|^{2}\left(\varphi^{+}\right)^{p-2}>0$.

Now, we show (7). Assume that there exists $u$ such that

$$
\frac{d}{d \lambda} \mathcal{L}\left(\lambda_{+}\right) \varphi^{+}=-(-\Delta)^{-1} m(x)(1-K) \varphi^{+}=u-(-\Delta)^{-1} m(x) \lambda_{+}(1-K) u,
$$


then

$$
\left(-\Delta-\lambda_{+}(m) m(x)\right) u=-(1-K) m(x) \varphi^{+},
$$

and so, multiplying by $\varphi^{+}$we get a contradiction using Lemma 2.1 .

Now, we can apply the Crandall-Rabinowitz Theorem [15] and conclude that there exists $\delta>0$ such that in a neighborhood of $\left(\lambda_{+}, 0\right)$ the nontrivial solutions of (1) are of the form

$$
\begin{aligned}
& u(s)=s \varphi^{+}+s^{2} \varphi_{2}+s^{3} \varphi_{3}+o\left(s^{3}\right), \\
& \lambda(s)=\lambda_{+}+s \lambda_{1}+s^{2} \lambda_{2}+o\left(s^{2}\right) .
\end{aligned}
$$

Introducing these terms in (1), using (8) and a Taylor expression of the function $1 /(1+u(s))$, we get

$$
\left(-\Delta-\lambda_{+}(m) m(x)\right) \varphi_{2}=\lambda_{+} m(x)\left(\varphi^{+}\right)^{2}(K \mp 1)+\lambda_{1} m(x)(1-K) \varphi^{+},
$$

and so,

$$
\lambda_{1}=-\frac{\lambda_{+}(K \mp 1)}{1-K} \frac{\int_{\Omega} m(x)\left(\varphi^{+}\right)^{3}}{\int_{\Omega} m(x)\left(\varphi^{+}\right)^{2}} .
$$

Observe that in the particular case $f=f_{2}$ and $K=-1, \lambda_{1}=0$, and so we have to calculate $\lambda_{2}$. It can be proved that

$$
\lambda_{2}=-\frac{\lambda_{+}}{2} \frac{\int_{\Omega} m(x)\left(\varphi^{+}\right)^{4}}{\int_{\Omega} m(x)\left(\varphi^{+}\right)^{2}} .
$$

From (9) and (10), we conclude the paragraph (2) of Theorem 1.1. Analogously it can be treated the case $\lambda_{-}$.

\subsection{Non-existence results}

Lemma 2.2. Assume $f=f_{1}$ and $K>1$ or $f=f_{2}$ and $K<1$. Then, there exists $\lambda^{*}>0$ such that for $\lambda \geq \lambda^{*}$ (1) does not have positive solutions.

Proof: Assume $f=f_{1}$ and $K>1$. Firstly observe that

$$
h(x):=x\left(\frac{K}{1+x}-1\right) \leq(\sqrt{K}-1)^{2}, \quad \forall x \geq 0 .
$$


Let $u$ be a positive solution of (1). Then, using the monotony of the principal eigenvalue with respect to the domain and (11) we get

$$
\begin{aligned}
0 & =\sigma_{1}\left[-\Delta-\lambda m(x)(1-K)-\lambda m(x) u\left(\frac{K}{1+u}-1\right)\right]< \\
& <\sigma_{1}^{M_{-}}\left[-\Delta-\lambda m(x)\left((1-K)+(\sqrt{K}-1)^{2}\right)\right]= \\
& =\sigma_{1}^{M_{-}}[-\Delta-\lambda m(x) 2(1-\sqrt{K})],
\end{aligned}
$$

which is an absurdum for $\lambda$ large.

Now, assume $f=f_{2}$ and $K<1$. In this case,

$$
\begin{aligned}
& x\left(\frac{K}{1+x}+1\right) \geq 0, \quad \text { if } K \geq-1, \quad \forall x \geq 0, \\
& x\left(\frac{K}{1+x}+1\right) \geq-(\sqrt{-K}-1)^{2}, \quad \text { if } K<-1, \quad \forall x \geq 0 .
\end{aligned}
$$

So, if $-1 \leq K<1$ we have

$0=\sigma_{1}\left[-\Delta-\lambda m(x)(1-K)-\lambda m(x) u\left(\frac{K}{1+u}+1\right)\right]<\sigma_{1}^{M_{+}}[-\Delta-\lambda m(x)(1-K)] ;$

on the other hand, for $K<-1$,

$0=\sigma_{1}\left[-\Delta-\lambda m(x)(1-K)-\lambda m(x) u\left(\frac{K}{1+u}+1\right)\right]<\sigma_{1}^{M_{+}}[-\Delta-\lambda m(x) 2 \sqrt{-K}]$,

in both cases a contradiction for large $\lambda$.

\subsection{Multiplicity results}

To obtain multiplicity results, we include (1) in the more general equation

$$
\begin{cases}-\Delta u=\mu m(x)(1-K) u+\lambda m(x) g(u) & \text { in } \Omega, \\ u=0 & \text { on } \partial \Omega,\end{cases}
$$

where $g$ satisfies

$\left(H_{g}\right) \quad g(0)=g^{\prime}(0)=0, \quad g^{\prime \prime}(u)<0, \quad \lim _{s \rightarrow+\infty} \frac{g(s)}{s^{2}}=\beta<0$.

Problem (12) has attracted a great deal of attention during last years (see for example [1], [3], [5], [6], [18] and [24]) when $m \equiv 1$ in the first term on the right-hand side of (12) and in [11], [12] and [13] with the right-hand side of the form $\mu h(x) u+g(x) u^{p}$ and restrictive conditions on $h$ and $g$ which are not satisfied in our case. In [16] was proved (see Fig. 3):

Proposition 2.1. Assume that $g$ satisfies $\left(H_{g}\right),(6), K \neq 1$ and fix $\lambda>0$. Denote by

$$
\Lambda_{+}:=\lambda_{+}(m(x)(1-K)), \quad \Lambda_{-}:=\lambda_{-}(m(x)(1-K)) .
$$


Then, (12) possesses a positive solution if $\mu>\Lambda_{-}$. Moreover, from the trivial solution $u=0$ emanate two unbounded in $\mathbb{R} \times C(\bar{\Omega})$ continua of positive solutions $\mathcal{C}_{+}:=\left\{\left(\mu, u_{\mu}\right)\right\}$ and $\mathcal{C}_{-}:=\left\{\left(\mu, w_{\mu}\right)\right\}$ at $\mu=\Lambda_{+}$and $\mu=\Lambda_{-}$, respectively. Both continua bifurcate to the right and $\Pi\left(\mathcal{C}_{-}\right) \supset\left(\Lambda_{-},+\infty\right)$, $\Pi\left(\mathcal{C}_{+}\right)=\left(\Lambda_{+},+\infty\right)$. Finally, for $\mu>\Lambda_{+}, u_{\mu}$ is linearly asymptotically stable and $u_{\mu} \neq w_{\mu}$.

Remark 2.1. Observe that for $K<1$,

$$
\Lambda_{+}=\lambda_{+} \quad \text { and } \quad \Lambda_{-}=\lambda_{-},
$$

and for $K>1$,

$$
\Lambda_{+}=\lambda_{-} \quad \text { and } \quad \Lambda_{-}=\lambda_{+} .
$$

Indeed, for example for $K>1$, it follows that

$\Lambda_{+}=\lambda_{+}(m(x)(1-K))=\frac{\lambda_{+}(-m(x))}{K-1}=\frac{-\lambda_{-}(m(x))}{K-1}=\frac{\lambda_{-}(m(x))}{1-K}=\lambda_{-}$.

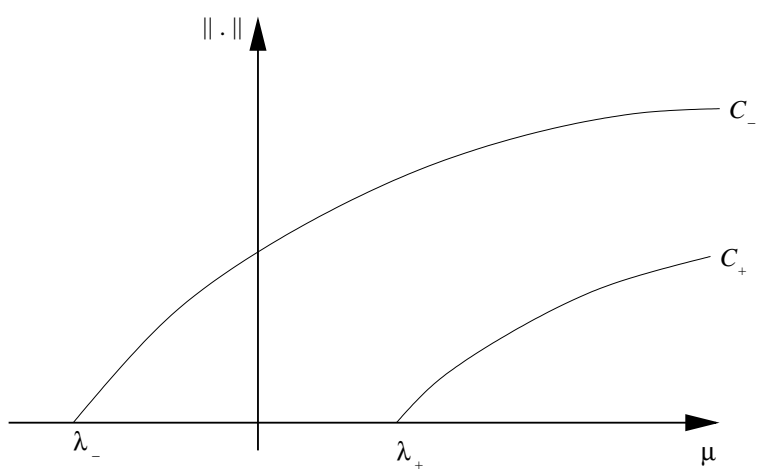

Figure 3. Bifurcation diagram for (12) and $K<1$.

\subsection{Proof of Theorem 1.1:}

Before proving the result, we generalize a well-known result for $m \equiv 1$. The proof is coming from [8].

Lemma 2.3. Assume that $f$ is a regular function and $f(0)=0$. Let $u_{0}$ be a positive solution of (1) such that $f\left(u_{0}\right)>0$, it holds: 
(1) If $f^{\prime \prime}\left(u_{0}\right)<0$, then $u_{0}$ is linearly asymptotically stable.

(2) If $f^{\prime \prime}\left(u_{0}\right)>0$, then $u_{0}$ is unstable.

Proof: We have to calculate the sign of the eigenvalue $\sigma_{1}[-\Delta-$ $\left.\lambda m(x) f^{\prime}\left(u_{0}\right)\right]$. Take $\psi:=f\left(u_{0}\right)>0$, then

$$
\left(-\Delta-\lambda m(x) f^{\prime}\left(u_{0}\right)\right) \psi=-f^{\prime \prime}\left(u_{0}\right)\left|\nabla u_{0}\right|^{2} .
$$

So, if $f$ is concave (resp. convex) the function $\psi$ is a supersolution (resp. subsolution) of $-\Delta-\lambda m(x) f^{\prime}\left(u_{0}\right)$, and then (see [23]) $\sigma_{1}[-\Delta-$ $\left.\lambda m(x) f^{\prime}\left(u_{0}\right)\right]>0($ resp. $<0)$.

The following result is proved in Theorem 3.4 of [3] and provides us with a priori bounds for the positive solutions of (1).

Lemma 2.4. Assume (6). If $(\lambda, u)$ is a positive solution of (1) and $\lambda \in J$, where $J$ is a compact subset such that $J \subset(0, \infty)$, then there exists a positive constant $C$ (independent from $\lambda$ ) such that

$$
\|u\|_{\infty} \leq C
$$

Finally, the following result is proved in [17].

Lemma 2.5. Assume that $\Sigma \subset I \times C_{0}^{2}(\Omega), I \subset \mathbb{R}$ an interval, is a connected set of positive solutions of $(1)$. Consider $\bar{u}: I \mapsto C_{0}^{2}(\Omega)$ a continuous map of supersolution for each $\lambda \in I$, but not a solution. If $u_{0}<\bar{u}\left(\lambda_{0}\right)$ for some $\left(\lambda_{0}, u_{0}\right) \in \Sigma$, then $u<\bar{u}(\lambda)$ for all $(\lambda, u) \in \Sigma$.

We are ready to prove the result. By subsec. 2.1 we know that there exists bifurcation from the trivial solution at $\lambda=\lambda_{+}$or $\lambda=\lambda_{-}$when $K<1$ or $K>1$, respectively. Moreover, we can apply Theorem 6.4 .3 of [25], and conclude that from $\lambda=\lambda_{+}$or $\lambda=\lambda_{-}$bifurcates an unbounded continuum $\mathcal{C}$ of positive solutions of (1). We would like to remark that the a detailed proof that $\mathcal{C}$ is unbounded and it does not satisfy the other alternatives of the above mentioned result will be presented elsewhere.

Now assume $f=f_{1}$ and $K<1$. It is clear that

$$
\bar{u}:=\sqrt{1-K}
$$

is a supersolution of (1). So, we can apply Lemma 2.5 (taking $\lambda_{0}=\lambda_{+}$) and conclude that

$$
\text { for all }\left(\lambda, u_{\lambda}\right) \in \mathcal{C} \text {, we have that } u_{\lambda}<\sqrt{1-K} .
$$

Moreover, $f_{1}\left(u_{\lambda}\right)>0$ and $f_{1}^{\prime \prime}\left(u_{\lambda}\right)<0$, and so by Lemma 2.3 we get that $u_{\lambda}$ is linearly asymptotically stable. 
Now, we are going to apply Proposition 2.1. Recall that in this case $\Lambda_{+}=\lambda_{+}$and $\Lambda_{-}=\lambda_{-}$. Taking as

$$
g(u)=u^{2}\left(\frac{K}{1+u}-1\right)
$$

we obtain a positive solution for $\mu=\lambda$ and $\lambda \in\left(0, \lambda_{+}\right]$and at least two positive solutions for $\lambda>\lambda_{+}$.

Similarly, it can be considered the case $f=f_{2}$ and $K>1$. Indeed, we only have to write $\mu m(x)(1-K) u+\lambda m(x) u^{2}(K /(1+u)+1)$ as

$$
\mu(-m(x))(K-1) u+\lambda(-m(x)) u^{2}(-K /(1+u)-1) .
$$

Observe that $g(u)=u^{2}(-K /(1+u)-1)$ satisfies $\left(H_{g}\right)$ for $K>-1$, and so, Proposition 2.1 is true for

$$
\Lambda_{+}=\lambda_{+}(-m(x)(K-1)), \quad \text { and } \quad \Lambda_{-}=\lambda_{-}(-m(x)(K-1)) .
$$

And, since $K>1$ it follows by Remark 2.1 that $\Lambda_{+}=\lambda_{-}$.

The paragraphs (4) and (5) follow easily from the existence of $\mathcal{C}$ and Lemmas 2.2 and 2.4 .

In order to prove paragraph (6), assume that there exist a sequence $\left(\lambda_{n}, u_{n}\right)_{n \in \mathbf{N}}$ of positive solution with $\lambda_{n} \rightarrow 0$ and $\left\|u_{n}\right\|_{\infty} \leq C$ for some $C>0$. Since there does not exist positive solution of (1) for $\lambda=0$, we obtain that $\left\|u_{n}\right\|_{\infty} \rightarrow 0$. We claim that this is impossible. Indeed, we define

$$
w_{n}=\frac{u_{n}}{\left\|u_{n}\right\|_{\infty}},
$$

then $w_{n}$ is uniformly bounded and, by passing to a suitable sequence again denoted by $w_{n}, w_{n} \rightarrow w^{*}$ as $n \rightarrow \infty$ for some $w^{*} \in C(\bar{\Omega})$ with $\left\|w^{*}\right\|_{\infty}=1$. But,

$$
-\Delta w_{n}=\lambda_{n} m(x) \frac{f\left(u_{n}\right)}{\left\|u_{n}\right\|_{\infty}},
$$

and so $-\Delta w^{*}=0$, which is an absurd. This concludes the proof.

\section{The particular case $K=1$}

In this case, the bifurcation from the trivial solution disappears. Consider

$$
\begin{cases}-\Delta u=\mu u+\lambda m(x) g(u) & \text { in } \Omega, \\ u=0 & \text { on } \partial \Omega,\end{cases}
$$

where

$$
g(u)=u^{2}\left(\frac{1}{1+u}-1\right) \quad \text { or } \quad g(u)=u^{2}\left(\frac{1}{1+u}+1\right) .
$$


Proposition 3.1. There exists a positive solution of (14) for $\mu=0$.

In particular, for all $\lambda>0$ there exists a positive solution of (1).

Proof: It easy to prove that this problem is in the setting of some works, see for example [3] and references therein, and then there exists an unbounded continuum $\mathcal{S}$ of positive solutions of (14) bifurcating from $\mu=\sigma_{1}[-\Delta]$ and it satisfies that $\Pi(\mathcal{S}) \supset\left(-\infty, \sigma_{1}[-\Delta]\right)$ (see Theorem 7.1 in [3]). This concludes the proof.

\section{References}

1. S. Alama, G. Tarantello, On the semilinear elliptic equations with indefinite nonlinearities, Calc. Var. Partial Differential Equations, 1 (1993), 439-475.

2. H. Amann, Fixed point equations and nonlinear eigenvalue problems in ordered Banach spaces, SIAM Rev., 18 (1976), 620-709.

3. H. Amann, J. López-Gómez, A priori bounds and multiple solutions for superlinear indefinite elliptic problems, J. Differential Equations, 146 (1998), 336-374.

4. A. Ambrosetti, P. Hess, Positive solutions of asymptotically linear elliptic eigenvalue problems, J. Math. Anal. Appl., 73 (1980), 411-422.

5. H. Berestycki, I. Capuzzo-Dolcetta, L. Nirenberg, Superlinear indefinite problems and nonlinear Liouville theorems, Topol. Methods Nonlinear Anal., 4 (1994), 59-78.

6. H. Berestycki, I. Capuzzo-Dolcetta, L. Nirenberg, Variational methods for indefinite superlinear homogeneous elliptic problems, NoDEA Nonlinear Differential Equations Appl., 2 (1995), 553-572.

7. J. Blat, K. J. Brown, Global bifurcation of positive solutions in some system of elliptic equations, SIAM J. Math. Anal., 17 (1986) 1339-1353.

8. K. J. Brown, P. Hess, Stability and uniqueness of positive solutions for a semi-linear elliptic boundary value problem, Differential Integral Equations, 3 (1990), 201-207.

9. K. J. Brown, B. Ko, The existence of positive solutions for a class of indefinite weight semilinear elliptic boundary value problem, Nonlinear Anal., 39 (2000), 587-597.

10. N. Y. Các, J. A. Gatica, Y. Li, Positive solutions to semilinear problems with coefficient that changes sign, Nonlinear Anal., 37 (1999), 501-510.

11. S. Cano-Casanova, Compact components of positive solutions for superlinear indefinite elliptic problems of mixed type, to appear in Topol. Meth. Nonl. Anal.

12. S. Cano-Casanova, J. López-Gómez, M. Molina-Meyer, Isolas, submitted.

13. S. Cingolani, J. L. Gámez, Positive solutions of a semilinear elliptic equation on $\mathbb{R}^{N}$ with indefinite nonlinearity, Adv. Differential Equations, 1 (1996), 773-791.

14. P. Clément, G. Sweers, Existence and multiplicity results for a semilinear 
elliptic eigenvalue problem, Annal. Scuola Norm. Sup. Pisa, Cl Sci., 4 (1987), 97-121.

15. M. G. Crandall, P. H. Rabinowitz, Bifurcation from simple eigenvalues, J. Funct. Anal., 8 (1971), 321-340.

16. M. Delgado, A. Suárez, On the existence and multiplicity of positive solutions for some indefinite nonlinear eigenvalue problem, to appear in Proc. Am. Math. Soc.

17. J. L. Gámez, Sub- and super-solutions in bifurcation problems, Nonlinear Anal., 28 (1997), 625-632.

18. R. Gómez-Reñasco, J. López-Gómez, The effect of varying coefficients on the dynamics of a class of superlinear indefinite reaction-diffusion equations, J. Differential Equations, 167 (2000), 36-72.

19. P. Hess, T. Kato, On some linear and nonlinear eigenvalue problems with an indefinite weight function, Comm. Partial Differential Equations, 5 (1980), 999-1030.

20. P. Korman, J. Shi, New exact multiplicity results with an application to a population model, Proc. Royal Soc. Edin., 131A (2001), 1167-1182.

21. P. L. Lions, On the existence of positive solutions of semilinear elliptic equations, SIAM Rev., 24 (1982), 441-467.

22. Z. Liu, Positive solutions of a class of nonlinear elliptic eigenvalue problems, Math. Z., 242 (2002), 663-686.

23. J. López-Gómez, The maximum principle and the existence of principal eigenvalues for some linear weighted boundary value problems, J. Differential Equations, 127 (1996), 263-294.

24. J. López-Gómez, On the existence of positive solutions for some indefinite superlinear elliptic problems, Comm. Partial Differential Equations, 22 (1997), 1787-1804.

25. J. López-Gómez, Spectral Theory and Nonlinear Functional Analysis, Research Notes in Mathematics 426, CRC Press, Boca Raton, 2001.

26. T. Ouyang, J. Shi, Exact multiplicity of possitive solutions for a class of semilinear, Parts I and II, J. Differential Equations, 146 (1998), 121-156 and 158 (1999), 94-151. 